\title{
A case report with a literature review: cerebral meningioma diagnosed by convulsion and consciousness disorder on initiating hemodialysis
}

Miki Uwatoko', Koki Tokunaga ${ }^{1 *}$, Mako Kawano ${ }^{1}$, Masahisa Kamimura', Yutaro Ibi ${ }^{1}$, Masayuki Otsuka', Masato Minami ${ }^{1}$, Haruhito Yoshimine ${ }^{1}$, Fujio Hamada ${ }^{2}$ and Akio Ido ${ }^{1}$

\begin{abstract}
Background: Neurological symptoms sometimes occur in hemodialysis patients, with causes including cerebral infarction, cerebral hemorrhaging, meningitis, and encephalitis. Dialysis disequilibrium syndrome (DDS) is widely known as a complication of hemodialysis and is typically encountered in severe uremic patients newly started on hemodialysis. The pathogenesis of DDS is thought to be brain edema that manifests as neurological symptoms, including headache, nausea, confusion, seizures, and coma. However, the relationship between brain tumors and neurological manifestations during hemodialysis is poorly understood.

Case presentation: The patient was a 55-year-old man with severe renal dysfunction and uremia symptoms. Blood chemistry showed severe azotemia and acidosis. The patient was placed on short-duration hemodialysis $(2 \mathrm{~h})$ with a relatively small surface area, low blood flow $(100 \mathrm{~mL} / \mathrm{min})$, and intradialytic glycerol infusion to prevent DDS. However, after his first hemodialysis treatment, he complained of disturbed consciousness. We diagnosed his neurological condition as DDS and observed the progress carefully. The next morning, his symptoms had completely resolved, so the patient was started on his second hemodialysis session with the same conditions. However, $2 \mathrm{~h}$ after starting the second hemodialysis session, he suffered convulsions accompanied by impaired consciousness. Brain computed tomography (CT) revealed a convexity meningioma and cerebral edema with a midline shift. Before starting the hemodialysis, he had shown no history of seizures, morning headache, or other neurological symptoms. In this case, meningioma was diagnosed based on an increase in the intracranial pressure which occurred after the initiation of hemodialysis.
\end{abstract}

Conclusion: We report this suggestive case to prompt physicians to consider the potential effect of hemodialysis introduction on the brain pressure.

Keywords: Introduction of hemodialysis, Azotemia, Dialysis disequilibrium syndromes, Cerebral hypertension, Occult brain tumor

\footnotetext{
* Correspondence: kokit@m2.kufm.kagoshima-u.ac.jp

${ }^{1}$ Digestive and Lifestyle Diseases, Kagoshima University Graduate School of

Medical and Dental Sciences, 8-35-1 Sakuragaoka, Kagoshima-shi, Kagoshima,

Japan

Full list of author information is available at the end of the article
}

(C) The Author(s). 2020 Open Access This article is distributed under the terms of the Creative Commons Attribution 4.0 International License (http://creativecommons.org/licenses/by/4.0/), which permits unrestricted use, distribution, and reproduction in any medium, provided you give appropriate credit to the original author(s) and the source, provide a link to the Creative Commons license, and indicate if changes were made. The Creative Commons Public Domain Dedication waiver (http://creativecommons.org/publicdomain/zero/1.0/) applies to the data made available in this article, unless otherwise stated. 


\section{Background}

Dialysis disequilibrium syndrome (DDS) refers to several neurological adverse effects that can occur as complications when introducing hemodialysis (HD). We herein report a severely uremic patient who developed DDS-induced cerebral edema and was diagnosed with meningioma. In this case, meningioma was diagnosed based on an increase in the intracranial pressure (ICP) which occurred after the initiation of hemodialysis.

DDS is a clinical phenomenon attributed to cerebral edema that occurs during or following HD. The precise incidence of DDS is unknown but appears to show a decreasing trend, most likely because patients today are set to start dialysis at lower urea concentrations than in the past and initiate HD using relatively advanced dialysis technology, with lower flow rates for both the blood and dialysate, a smaller dialysate volume, and more frequent dialysis sessions [1]. In the present case, an ICP surge induced after the initiation of HD increased the pressure to a greater degree than expected because of the existence of occult meningioma in addition to severe uremia, which also carried a risk of causing DDS.

This is the first reported case of DDS induced by meningioma in a patient that developed on the initiation of hemodialysis. We review the relevant literature concerning the association of DDS and ICP.

\section{Case presentation}

A 55-year-old man with obstructive nephropathy and dehydration was admitted for severe azotemia. He had a known history of hypertension (treated for 5 years) and benign prostatic hyperplasia (BPH). Despite having an appetite, he had been unable to eat or drink to satisfaction because of nausea for the past month. His urine volume and body weight had gradually decreased before admission in our hospital. On admission, he was very sick with progressive tachypnea, respiratory distress, and vomiting. And his urine volume decreased from 1 month ago. His serum creatinine and blood urea nitrogen were very high. We diagnosed the patient to have chronic kidney failure due to nephrosclerosis, and the acute exacerbation of chronic renal failure was caused by ureteral obstruction due to $\mathrm{BPH}$ and bladder hypertrophy.

Initial laboratory results are presented in Table 1 . The findings from blood investigations were as follows: hemoglobin $(\mathrm{Hb}), 5.8 \mathrm{~g} / \mathrm{dL}$; hematocrit $(\mathrm{Ht}), 17.6 \%$; so$\operatorname{dium}(\mathrm{Na}), 150 \mathrm{mmol} / \mathrm{L}$; potassium $(\mathrm{K}), 7.3 \mathrm{mmol} / \mathrm{L}$; blood urea nitrogen (BUN), $287.2 \mathrm{mg} / \mathrm{dL}$; creatinine (Cr), $32.98 \mathrm{mg} / \mathrm{dL}$; C-reactive protein (CRP), $0.54 \mathrm{mg} / \mathrm{dL}$; and blood glucose, $126 \mathrm{mg} / \mathrm{dL}$. Arterial blood gas analyses showed a $\mathrm{pH}$ of 7.0, $\mathrm{PaCO}_{2} 15.1 \mathrm{mmHg}, \mathrm{PaO}_{2} 57.6$ $\mathrm{mmHg}, \mathrm{HCO}_{3} 3.7 \mathrm{mmol}$, and lactate $3.3 \mathrm{mmol}$, which was consistent with high-anion-gap metabolic acidosis with respiratory compensation. Renal anemia was

Table 1 Laboratory findings on admission

\begin{tabular}{|c|c|c|c|c|c|}
\hline \multicolumn{2}{|c|}{ Blood gas analysis (room air) } & \multicolumn{2}{|l|}{ Chemistry } & \multicolumn{2}{|l|}{ Immunological tests } \\
\hline$\overline{\mathrm{pH}}$ & 7.004 & Total protein & $6.6 \mathrm{~g} / \mathrm{dL}$ & $\overline{\mathrm{ANA}}$ & $<40$ \\
\hline $\mathrm{pCO}_{2}$ & 15.1 & Albumin & $3.3 \mathrm{~g} / \mathrm{dL}$ & MPO-ANCA & $0.7 \mathrm{IU} / \mathrm{mL}$ \\
\hline $\mathrm{PO} 2$ & 57.6 & BUN & $287.2 \mathrm{mg} / \mathrm{dL}$ & PR3-ANCA & $0.7 \mathrm{IU} / \mathrm{mL}$ \\
\hline $\mathrm{HCO} 3$ & 3.7 & Creatinine & $32.98 \mathrm{mg} / \mathrm{dL}$ & Anti-GBM antibody & $2.2 \mathrm{IU} / \mathrm{mL}$ \\
\hline Base excess & -25.3 & Uric acid & $11.9 \mathrm{mg} / \mathrm{dL}$ & Intact-PTH & $220 \mathrm{pg} / \mathrm{dL}$ \\
\hline Anion gap & 39.2 & Sodium & $150 \mathrm{mEq} / \mathrm{L}$ & PSA & $2.8 \mathrm{ng} / \mathrm{mL}$ \\
\hline \multirow[t]{2}{*}{ Lactate } & 3.3 & Potassium & $7.3 \mathrm{mEq} / \mathrm{L}$ & & \\
\hline & & Chloride & $114 \mathrm{mEq} / \mathrm{L}$ & & \\
\hline Blood & & Calcium & $6.1 \mathrm{mg} / \mathrm{dL}$ & & \\
\hline WBC count & $9890 / \mu \mathrm{L}$ & Phosphorus & $14.9 \mathrm{mg} / \mathrm{dL}$ & & \\
\hline RBC count & $197 \times 10^{4} / \mu \mathrm{L}$ & AST & $17 \mathrm{U} / \mathrm{L}$ & & \\
\hline Hemoglobin & $5.8 \mathrm{~g} / \mathrm{dL}$ & $\mathrm{ALT}$ & $22 \mathrm{U} / \mathrm{L}$ & & \\
\hline Hematocrit & $17.6 \%$ & $\mathrm{LDH}$ & $544 \mathrm{U} / \mathrm{L}$ & & \\
\hline MCV & $89.3 \mathrm{fL}$ & $\mathrm{Fe}$ & $166 \mu \mathrm{g} / \mathrm{dL}$ & & \\
\hline $\mathrm{MCHC}$ & $33 \%$ & Ferritin & 736 ng/mL & & \\
\hline \multirow[t]{3}{*}{ Platelet count } & $18.8 \times 10^{4} / \mu \mathrm{L}$ & CRP & $0.54 \mathrm{mg} / \mathrm{dL}$ & & \\
\hline & & Glucose & $126 \mathrm{mg} / \mathrm{dL}$ & & \\
\hline & & $\mathrm{HbA1c}$ & $5.1 \%$ & & \\
\hline
\end{tabular}

ANCA antineutrophil cytoplasmic antibody, HbA1c hemoglobin A1c, MPO myeloperoxidase, $P R$-3 proteinase-3, PSA prostate-specific antigen, $P T H$ parathyroid hormone, ANA antinuclear antibody, GBM glomerular basement membrane 
suspected because of his marked anemia and normocytic positive pigmented anemia without iron deficiency.

The patient's renal dysfunction was severe, with metabolic acidosis accompanied by an elevated anion gap and metabolic alkalosis, thought to be due to vomiting. The inflammatory response was reduced, and vasculitis markers able to induce rapidly progressive glomerulonephritis were negative. His changes in $\mathrm{P}, \mathrm{Ca}$, and intact PTH values were strongly suspected to be the result of chronic renal impairment, and the presence of anemia supported this notion. Hypoproteinemia from uremic malnutrition and hypernatremia due to dehydration were observed. A urinalysis was not conducted due to his anuria. Despite his hyperkalemia, he was not deemed to be in a severe medical condition because of no electrocardiogram abnormalities were observed.

On admission (day 1), he was alert without any other neurologic abnormalities. His height was $165 \mathrm{~cm}$, and his body weight was $54.6 \mathrm{~kg}$. He reported a weight loss of 10 $\mathrm{kg}$ over the past 2 months. His blood pressure was 118/60 $\mathrm{mmHg}$, body temperature was $36.6^{\circ} \mathrm{C}$, and pulse rate was $98 \mathrm{bpm}$. He was severely dehydrated with tachypnea, and his respiratory rate was 30/min with $\mathrm{SpO}_{2}$ 98\%. Conversation was intermittent due to tachypnea. His palpebral conjunctiva was pale. He had poor skin turgor, axillary and tongue drying, and no extremity edema. He had no history of headache, including morning headache. Chest radiography was normal. Abdominal ultrasonography (AUS) and chest-pelvic CT revealed severe bilateral hydronephrosis as well as bladder wall thickening due to $\mathrm{BPH}$. No urine accumulation in the bladder was observed. An echocardiogram revealed an ejection fraction $(\mathrm{EF})$ of $62 \%$, no wall motion disorder, and thickening of the myocardial wall with suspected hypertensive cardiomyopathy. An electrocardiogram showed a normal sinus rhythm and no QT time extension but a tented $\mathrm{T}$ wave.

After hospitalization, glucose-insulin therapy was started for his hyperkalemia. We diagnosed him with acute exacerbation of chronic renal failure due to ureteral obstruction and dehydration, where he had previously had nephrosclerosis. In his postrenal failure state, ureteral obstruction due to bladder wall thickening made it difficult to place a ureteral stent, so a right kidney fistula was made. After securing the outflow path of urine, follow-up was performed by increasing the replacement fluid volume in order to correct his dehydration caused by uremia. However, even after several hours of this treatment with the infusion of $2 \mathrm{~L}$ of normal bicarbonate solution, his anuria persisted.

Treatment with HD was therefore introduced to manage his refractory metabolic acidosis, hyperkalemia, and uremia symptoms and to ensure future transfusion spaces. We planned to perform HD every day for 3 days. The patient was placed on short-duration hemodialysis
$(2 \mathrm{~h})$ with a relatively small surface area (cellulose triacetate; membrane area, $1.0 \mathrm{~m}^{2}$ ), low dialysate flow rate ( $500 \mathrm{~mL} / \mathrm{min}$ without ultrafiltration), and low blood flow $(80-100 \mathrm{~mL} / \mathrm{min})$ to prevent DDS. Progress was noted after starting dialysis.

At the end of the initial 2-h dialysis session, his acidosis and tachypnea were relieved. However, although the patient had been alert before the initiation of HD, during the session, he became gradually obtunded, with a Glasgow Coma Scale (GCS) of 13/15 (E3V4M6). He did not complain of a headache or paralysis, and we thought that he had somehow developed DDS. A mild disturbance of consciousness appeared about $1 \mathrm{~h}$ after starting HD and lasted throughout the treatment and beyond the session. We followed him with replacement fluid, and his consciousness became clear again by the next morning. Therefore, his consciousness disorder was not considered contradictory to the symptoms of DDS.

Dialysis was started again the second day as planned. However, less than $2 \mathrm{~h}$ after starting HD, the patient developed sudden generalized tonic convulsion, a consciousness level of GCS 3/15 (E1V1M1), and respiratory arrest. We treated him with intravenous anticonvulsant and intubation to relieve his respiratory distress.

We stopped the dialysis session and performed head CT. It showed a brain tumor of $5 \mathrm{~cm}$ with diffuse cerebral edema (Fig. 1). Additional enhanced CT for tumor scrutiny resulted in a diagnosis of meningioma morphologically. The cerebral edema had been exacerbated by dialysis, inducing convulsions when the brain pressure had been increased due to occult meningioma.

We started treatment with mannitol ${ }^{\circ}$ to reduce his cerebral edema. We planned to respond with continuous hemodiafiltration (CHDF) if the anuria had persisted after the following day. However, continued systemic management by replacement fluid, transfusion, and mannitol infusion fortunately resulted in urine being released from day 4 of the disease. The patient's renal failure entered the diuretic phase, and gradually, his renal function ameliorated, after which dialysis became unnecessary.

With no new episodes of convulsions appearing, his consciousness and respiration condition gradually improved and returned to clear on day 8 . We continued treatment to protect his kidneys from anemia, acidosis, and electrolyte correction. Two weeks later, the edema of the bladder wall had decreased, so the nephrostomy was removed and changed to ureteral stent placement. The renal function ultimately improved and stabilized around BUN $70 \mathrm{mg} / \mathrm{dL}$ and Cr $5 \mathrm{mg} / \mathrm{dL}$ (Fig. 2). The patient was discharged without any neurological deficits, and no further neurological disturbances were noted during 1 year of follow-up. Transurethral prostatectomy was performed roughly 1.5 months later, and the ureteral stent was removed. In addition, brain tumor removal 

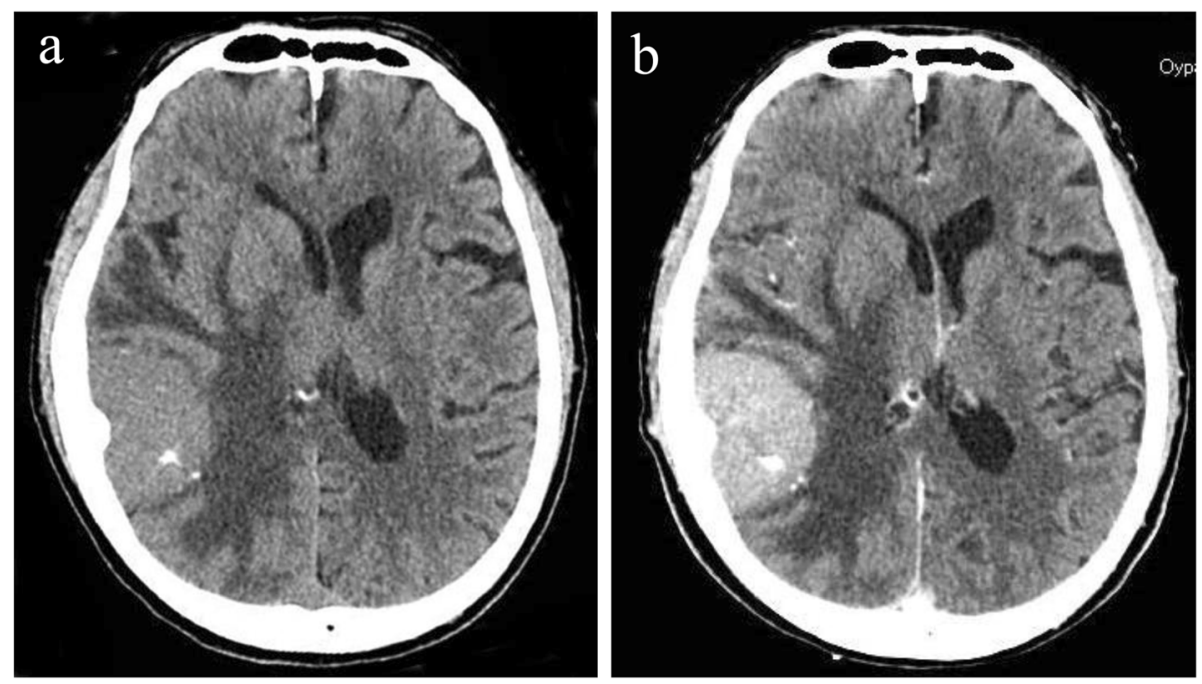

Fig. 1 Computed tomography image of the head. Approximately $5 \mathrm{~cm}$ of contrasting effect and brain tumor accompanied by calcification and midline shift by brain edema were recognized. a Plain. $\mathbf{b}$ Enhanced

surgery was performed 1 year later, and the lesion was pathologically confirmed to be meningioma. The patient's renal function has been consistently stable.

\section{Discussion}

In our case, we initiated HD in a severely uremic patient whose renal profile showed creatinine $32.98 \mathrm{mg} / \mathrm{dL}$ and urea $287.2 \mathrm{mg} / \mathrm{dL}$. We diagnosed the extremely high levels of uremia as being caused by ureteral obstruction due to $\mathrm{BPH}$ and bladder hypertrophy. The cause of acute renal failure is well known to be prostatic disease, including $\mathrm{BPH}$ [2]. Partial obstruction of the urinary bladder outlet, one of the major effects of $\mathrm{BPH}$, induces a significant increase in bladder mass and detrusor smooth muscle hypertrophy [3].

The patient's consciousness becomes gradually cloudy after HD. BUN increases the osmotic pressure, and the proper concentration of BUN helps to maintain the

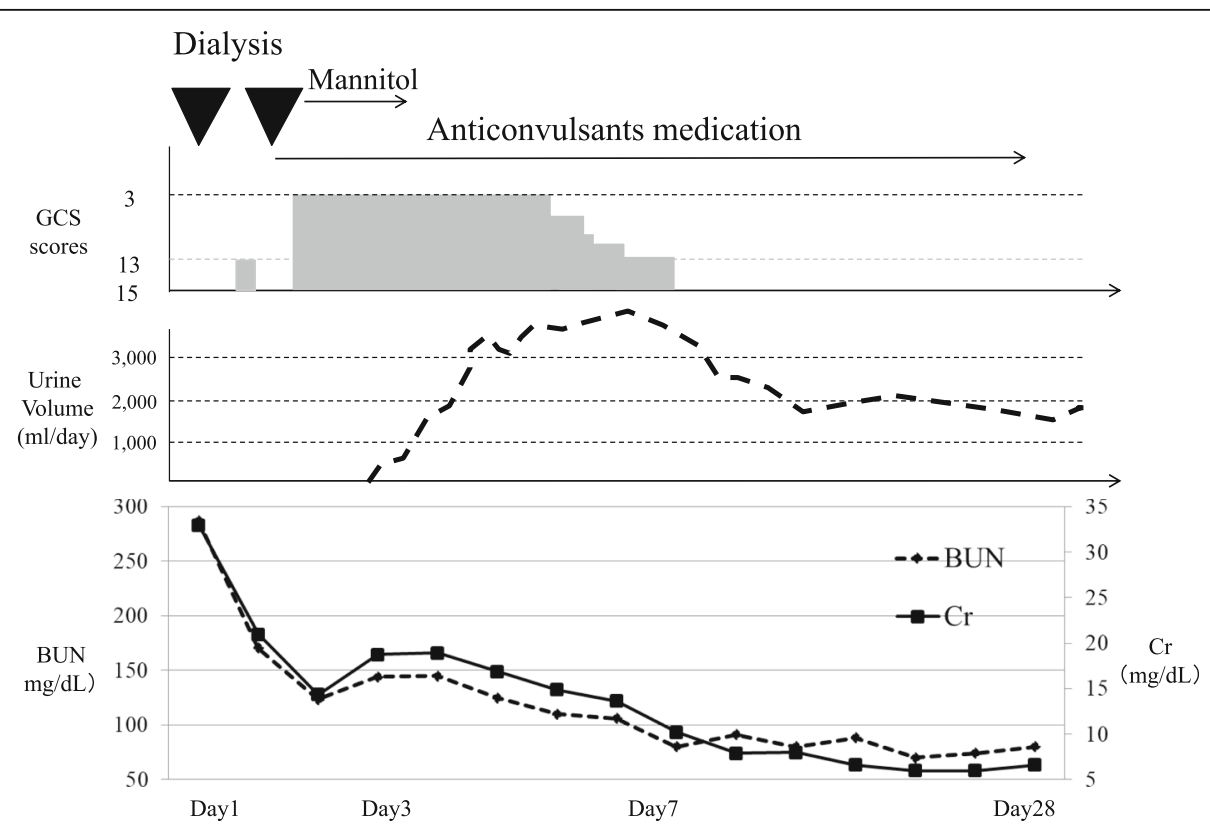

Fig. 2 Clinical course of the patients. After initial dialysis, GCS score reached 13 (E3 V4 M6), but it recovered the next day. Severe consciousness disturbance (E1 V1 M1) and convulsion occurred during the second dialysis. After that, dialysis did not need to be done and gradually improved his level of consciousness. GCS, Glasgow Coma Scale 
water balance in the body. Accordingly, a rapid change in the BUN concentration during HD results in a decline in osmotic pressure, causing water to move into the central nervous system [4]. We initially believed that he had developed DDS because of neurological symptoms observed.

DDS, defined initially in 1962, is an acute neurological complication of HD [5]. The pathogenesis remains debated and incompletely understood; however, several central hypotheses have emerged. The main theory is the "reverse urea effect," in which uremic substances, mainly urea nitrogen, are rapidly removed from the blood by HD initiation, resulting in an osmotic concentration gradient with the intracranial area via the bloodbrain barrier, resulting in cerebral edema and the development of DDS [6]. The fluctuation rate of BUN before and after dialysis increases as the pre-dialysis BUN value increases, and as a result, the osmotic pressure of the blood/central nervous system, which is the main cause of DDS onset, also increases. Patients with extremely high levels of BUN (above $175 \mathrm{mg} / \mathrm{dL}$ ) are at a higher risk of experiencing first-time HD-related complications, particularly DDS, than those with lower levels [7].

Other clinical risk factors for DDS are starting HD for the first time, severe uremia, an older or younger age (e.g., elderly or children), pre-existing neurological disorders, and severe metabolic acidosis [8, 9]. In addition, patients with pre-existing conditions predisposed to brain edema (sepsis, hypercapnia, malignant hypertension, hyponatremia, hypoglycemia, and hepatic encephalopathy) are much more susceptible to DDS than those without such conditions. It is important to identify patients at high risk for developing DDS [1]. According to several reports, during $\mathrm{HD}$, the plasma osmotic pressure level is reduced by $15-40 \mathrm{mOsm} / \mathrm{kg}$, but the difference in the osmotic pressure between the plasma and brain intracellular space ranges from 5 to $20 \mathrm{mOsm} / \mathrm{kg}$ because the intracellular osmotic pressure level drops slowly [10]. This phenomenon always results in an increase in the ICP [11]. The mean ICP starts to increase after the first hour of HD and remains high during HD. The ICP increase during HD initiation is measured directly in some acute or chronic renal failure patients with intracranial hemorrhaging or head trauma who undergo craniectomy or craniotomy $[9,11-16]$.

Some case reports have evaluated the presence of brain hypertension before and after HD in order to clarify the condition of cerebral edema. Furthermore, MRI findings of end-stage renal failure patients with severe azotemia show a reduction in the apparent diffusion coefficient after dialysis [17]. There have also been reports that the density is reduced in head CT and MRI after dialysis in patients with uremia. This change is considered to reflect the increase in the water content in the brain $[17,18]$. The predisposing factors for DDS in the present case were a high urea value, severe uremia symptoms, and severe metabolic acidosis. In our patient, even though we did not detect the brain tumor as a complication at the initial introduction of HD, we considered the patient to have a high risk of developing DDS due to the presence of multiple predicting factors. To prevent DDS, HD with reduced dialysis efficacy and a gradual urea reduction is recommended $[9,19,20]$. At the time of dialysis introduction, such patients should be placed on short-duration hemodialysis with low blood flow. The addition of mannitol, glycerol, or glucose to the dialysate or substitution of bicarbonate for acetate in the dialysate can also help reduce the risk of DDS. The initiation of continuous renal replacement therapy (CRRT) with a more gradual and stable clearance of urea should be considered as well in order to minimize the risk of DDS. Ideally, CRRT would have been used to treat the present patient. However, we unfortunately had no choice but to start short-duration HD due to a lack of adequate medical facilities.

Regarding the removal rate of BUN during effective dialysis to prevent DDS, some reports have suggested that the effective removal rate for patients with high BUN is less than $40 \%$ at $2 \mathrm{~h}$ or more [21]. However, there are reports of DDS developing in cases with BUN $>299 \mathrm{mg} / \mathrm{dL}$ even though the removal rate was $17 \%$ [7]. Therefore, it is necessary to pay close attention to the progress of dialysis in patients with markedly high BUN values who are prone to brain pressure elevation, even if DDS prevention measures are in place.

In the present patient, we tried to prevent DDS, but mild consciousness disturbance still occurred after the initial dialysis. Furthermore, despite the temporary relief of his symptoms, the second dialysis session the next day resulted in cramping, disturbance of consciousness, and breathing stoppage. At that time, the removal rate of BUN was relatively low at $28 \%$ (Table 2), and changes in blood sugar and serum sodium value considered unlikely to be a direct cause of the convulsions. Therefore, we considered the possibility that other pathological conditions had emerged and started scrutinizing the situation more carefully. Emergency head CT revealed a brain tumor and global cerebral edema around the tumor with a midline shift, and we diagnosed these as the causes of his convulsions.

Of note, we did not perform close scrutiny in this case when cloudy consciousness was first noted after the initial dialysis session. Since this patient started emergency dialysis with numerous factors associated with a risk of DDS, we did not vigorously search for other causes when neurological abnormal findings appeared. Regarding the subsequent course of this case, 1 week after the onset of convulsions and consciousness disturbance, his 
Table 2 Review of the literature on intracranial lesion in hemodialysis patients which were found to have dialysis disequilibrium syndrome

\begin{tabular}{lllllll}
\hline Reference & Age & Sex & Intracranial disorder & Period of HD & Type of blood purification & Outcome \\
\hline$[22]$ & 70 & Female & Neoplastic meningitis & 3 years & Maintenance HD & Recover \\
{$[23]$} & 5 & Male & Medulloblastoma & 5 years & Restart HD (PD $\rightarrow$ HD $\rightarrow$ transplant) & Recover \\
Our case & 55 & Male & Meningioma & First time & Initial HD (mild and short duration) & Recover
\end{tabular}

consciousness became clear. However, the midline shift remained even 2 weeks later, although the cerebral edema had been alleviated, and the improvement on CT images was considered insufficient. The midline shift finally disappeared after about a month, and the cerebral edema improved on $\mathrm{CT}$, being localized only around the tumor. After 1 month, no further improvements on images were noted (Fig. 3).

We examined whether the central nervous symptoms of this case were due to the changes in the brain pressure induced by dialysis or by the presence of the brain tumor itself in the course of dialysis withdrawal. The patient reported no history of early morning headache, which is said to be common when brain tumors are present. The patient was discharged without any neurological deficits, and no further neurological disturbances were noted after withdrawing HD. His neurological disturbances were noted during HD only. His nausea and vomiting, which he had complained of before starting HD, could also be explained generally by cerebral hypertension due to the brain tumor. However, his symptoms disappeared after we withdrew dialysis, and his renal function recovered. Therefore, his symptoms of nausea and vomiting were thought to be due to uremia. Given the above, we diagnosed the patient's condition as being due to the existence of an occult brain tumor; his brain pressure had already been high and overlapped with a further increase in the brain pressure after the initiation of dialysis, which thus led to the onset of cerebral hypertension.

\section{Literature review}

The cause of DDS is considered by increased intracranial pressure from the exacerbation of cerebral edema. The hyperpermeability of the blood-brain barrier is known to increase in intracranial pressure and meningitis, and vasculitis, CNS tumors, hemolytic uremic syndrome, and thrombotic thrombocytopenic purpura are at the risk of hyperpermeability [24]. It has been reported that newonset disease, such as intracranial bleeding, traumatic

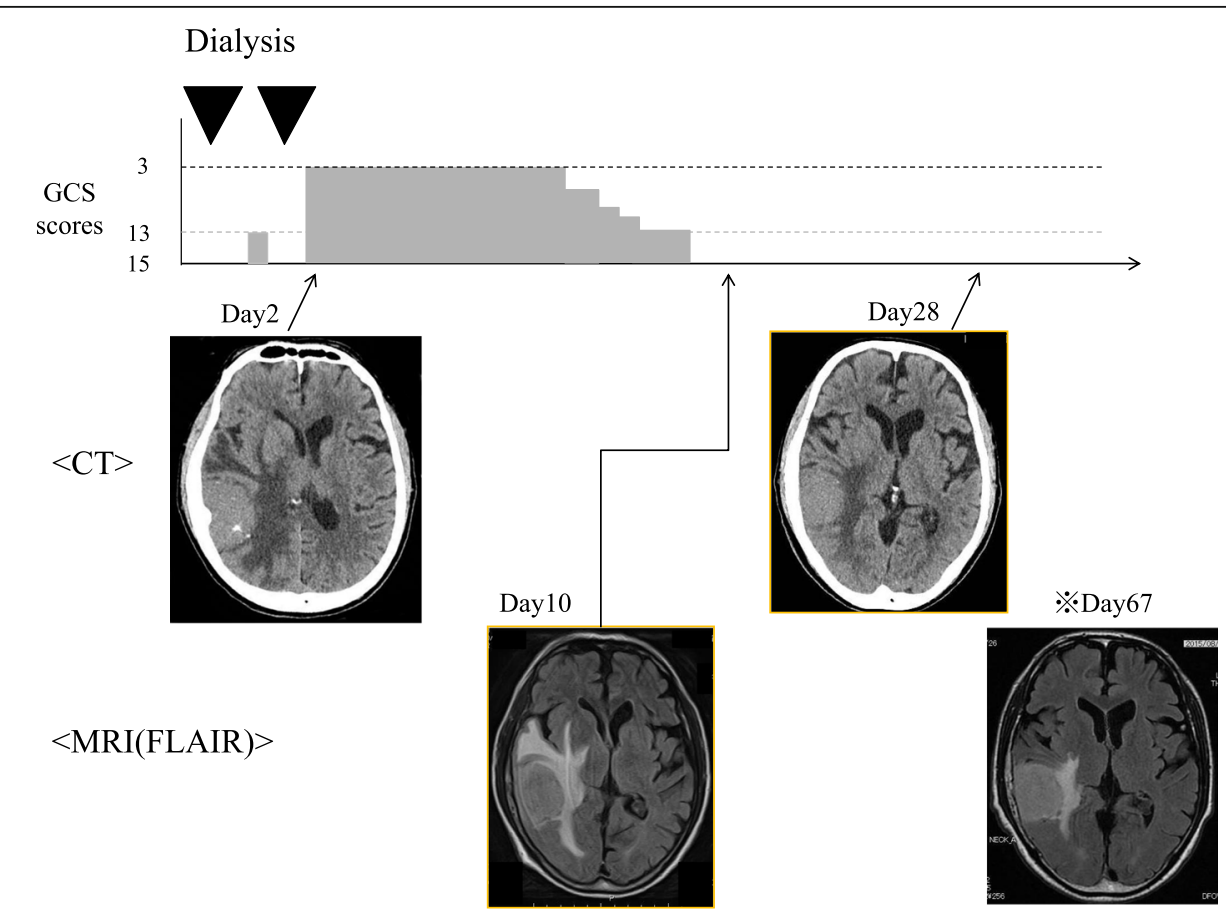

Fig. 3 Temporal changes on the image in abnormal cerebral edema and midline shift. Head CT scan on day 28 revealed improvement in midline shift. FLAIR image that was performed on day 67 showed marked improvement in brain edema around tumor on the right temporal region. FLAIR fluid-attenuated inversion recovery 
brain injury, neoplastic meningitis [22], and medulloblastoma [23], can cause DDS even in patients receiving maintenance HD.

Although there have been many cases in which DDS occurred after the treatment for intracranial lesions, there have been only three reports of DDS caused by dialysis as a key symptom by which asymptomatic intracranial tumors were diagnosed. The summary is shown in Table 2. With respect to meningitis that develops rapidly, DDS occurs during maintenance dialysis, but for slowly exacerbating neoplastic lesions, DDS occurs at the start of dialysis.

In the case reported by Dilena et al., a neurological symptom-suspected DDS has appeared from the beginning of the third dialysis session after the transplanted kidney loss.

\section{Conclusion}

We herein report a patient whose occult meningioma was diagnosed as being associated with severe DDS as a result of an increase in the ICP due to the initiation of HD. When DDS develops despite adequate preventive measures, scrutiny is necessary in order to evaluate the possibility of latent disease.

\section{Abbreviations}

AUS: Abdominal ultrasonography; BPH: Benign prostatic hyperplasia; BUN: Blood urea nitrogen; Ca: Calcium; CHDF: Continuous hemodiafiltration; Cr: Creatinine; CRRT: Continuous renal replacement therapy; CT: Computed tomography; DDS: Dialysis disequilibrium syndrome; GCS: Glasgow Coma Scale; HD: Hemodialysis; ICP: Intracranial pressure; MRI: Magnetic resonance imaging; P: Phosphorus; PTH: Parathyroid hormone

\section{Acknowledgements}

We thank Dr. Hiroyuki Deguchi who is currently giving medical treatment.

\section{Authors' contributions}

$\mathrm{MU}, \mathrm{MM}$, and $\mathrm{FH}$ took care of the patient. MU and KT prepared the manuscript. MK, MK, YI, MO, HY, and Al participated in the decision of treatment in the patient. All authors read and approved the final manuscript.

\section{Funding}

Not applicable.

\section{Availability of data and materials}

The data and materials were all included in the manuscript.

\section{Ethics approval and consent to participate}

According to the Ethical Guidelines for Medical and Health Research Involving Human Subjects in Japan, ethics approval is not necessary when submitting case reports.

\section{Consent for publication}

Written informed consent was obtained from the patient for the publication of this case report.

\section{Competing interests}

The authors declare that they have no competing interests.

\section{Author details}

'Digestive and Lifestyle Diseases, Kagoshima University Graduate School of Medical and Dental Sciences, 8-35-1 Sakuragaoka, Kagoshima-shi, Kagoshima, Japan. ${ }^{2}$ Division of Nephrology, Saiseikai Sendai Hospital, 2-46 Harada-chou, Satsumasenndai-shi, Kagoshima, Japan.
Received: 27 May 2019 Accepted: 28 January 2020

Published online: 09 April 2020

\section{References}

1. Arieff Al. Dialysis disequilibrium syndrome: current concepts on pathogenesis and prevention. Kidney Int. 1994;45(3):629-35.

2. Feest TG, Round A, Hamad S. Incidence of severe acute renal failure in adults: results of a community based study. BMJ. 1993;306(6876):481-3.

3. Bing W, Chang S, Hypolite JA, DiSanto ME, Zderic SA, Rolf L, et al. Obstructioninduced changes in urinary bladder smooth muscle contractility: a role of Rho kinase. Am J Physiol Ren Physiol. 2003:285(5):F990-7.

4. Rosen SM, O' Connor K, Shaldon S. Haemodialysis disequilibrium. Br Med J. 1964;2:672-5.

5. Kennedy $A C$, Linton $A L$, Eaton JC. Urea levels in cerebrospinal fluid after hemodialysis. Lancet. 1962;1:410-1.

6. Silver SM, DeSimone JA Jr, Smith DA, Stens RH. Dialysis disequilibrium syndrome (DDS) in the rat: role of the "reverse urea effect". Kidney Int. 1992;4:161-6.

7. Lopez-Almaraz E, Correa-Rotter R. Dialysis disequilibrium syndrome and other treatment complications of extreme uremia: a rare occurrence yet not vanished. Hemodail Int. 2008:12(3):301-6.

8. Grushkin CM, Korsch B, Fine RN. Hemodialysis in small children. JAMA. 1972; 221(8):869-73.

9. Esnault P, Lacroix G, Cungi P-J, D' Aranda E, Cotte J, Goutorbe P. Dialysis disequilibrium syndrome in neurointensive care unit: the benefit of intracranial pressure monitoring. Crit Care. 2012;16:472.

10. Gondo G, Fujitsu K, Kuwabara T, Mochimitsu Y, Ishikawa Y, Oda H, et al. Comparison of five modes of dialysis in neurosurgical patients with renal failure. Neurol Med Chir (Tokyo). 1989;29:1125-31.

11. Davenport A, Will EJ, Davison AM. Early changes in intracranial pressure during haemofiltration treatment in patients with grade 4 hepatic encephalopathy and acute oliguric renal failure. Nephrol Dial Transplant. 1990;5:192-8.

12. Lin CM, Lin JW, Tsai JT, Ko CP, Hung KS, Hung CC, et al. Intracranial pressure fluctuation during hemodialysis in renal failure patients with intracranial hemorrhage. Acta Neurochir Suppl. 2008;101:141-4.

13. Bertrand YM, Hermant A, Mahieu P, Roles J. Intracranial pressure changes in patients with head trauma during hemodialysis. Intensive Care Med. 1983;9:321-3

14. Yang K-C, Chang C-J, Hsieh C-T. Dialysis disequilibrium syndrome: the change of intracranial pressure. SM J Case Rep. 2015;2:1006.

15. Yoshida S, Tajika T, Yamasaki N, Tanikawa T, Kitamura K, Kudo K, et al. Dialysis disequilibrium syndrome in neurosurgical patients. Neurosurgery. 1987:20:716-21

16. Krane NK. Intracranial pressure measurement in a patient undergoing hemodialysis and peritoneal dialysis. Am J Kidney Dis. 1989;13:336-9.

17. Chen CL, Lai PH, Chou KJ, Lee PT, Chung HIM, Fang HC. A preliminary report of brain edema in patients with uremia at first hemodialysis: evaluation by diffusion-weighted MR imaging. Am J Neuroradiol. 2007:28(1):68-71.

18. Dettori P, La Grace G, Biasioli S, Chiaramonte S, Fabris A, Feriani M, et al. Changes of cerebral density in dialyzed patients. Neuroradiology. 1982;23(2):95-9

19. Zepeda-Orozco D, Quigley R. Dialysis disequilibrium syndrome. Pediatr Nephrol. 2012;27(12):2205-11.

20. Davenport A. Practical guidance for dialyzing a hemodialysis patient following acute brain injury. Hemodial Int. 2008;12(3):307-12.

21. Patel N, Dalal P, Panesar M. Dialysis disequilibrium syndrome: a narrative review. Semin Dial. 2008:21(5):493-8.

22. Tsuchida Y, Tanaka T, Ikarashi T, lino N, Kazama JJ, Narita I. Dialysis disequilibrium syndrome induced by neoplastic meningitis in a patient receiving maintenance hemodialysis. BMC Nephrol. 2013;14:255.

23. Dilena R, Paglialonga F, Barbieri S, Edefonti A. Medulloblastoma presenting as dialysis disequilibrium syndrome. Hemodial Int. 2011;15(Suppl 1):S64-7.

24. Mistry K. Dialysis disequilibrium syndrome prevention and management. Int J Nephrol Renov Dis. 2019:1269-77.

\section{Publisher's Note}

Springer Nature remains neutral with regard to jurisdictional claims in published maps and institutional affiliations. 